\title{
Identification and Expression Analysis of Multiple Ferric Chelate Reductases in Citrus junos
}

\author{
Li-Xiao Yao, Yong-Rui He, Hai-Fang Fan, Lan-Zhen Xu, Tian-Gang Lei, Xiu-Ping Zou, \\ Ai-Hong Peng, and Qiang Li \\ National Citrus Engineering Research Center, Citrus Research Institute, Southwest University, \\ Chongqing 400712, Peoples Republic of China \\ Shan-Chun Chen ${ }^{1}$ \\ National Center for Citrus Variety Improvement, Citrus Research Institute, Chinese Academy of \\ Agricultural Sciences, Chongqing 400712, Peoples Republic of China
}

\begin{abstract}
AdDitional Index words. FRO, tissue expression, iron deficiency
Abstract. Ferric chelate reductase (FRO) is a critical enzyme for iron absorption in strategy I plants, reducing Fe ${ }^{3+}$ to $\mathrm{Fe}^{2+}$. To identify $\mathrm{FRO}$ family genes in the local Citrus junos cultivar Ziyang Xiangcheng and to reveal their expression model, the citrus (Citrus sp.) genome was searched for homologies of the published sequence $C$ jFRO1. Five $F R O$ s were found, including $C j F R O 1$; these were named $C j F R O 2, C j F R O 3, C j F R O 4$, and $C j F R O 5$, respectively, and cloned via reverse transcription polymerase chain reaction (RT-PCR) and rapid amplification of cDNA ends (RACE) PCR. The deduced amino acid sequences of five $C j F R O$ s contained flavin adenine dinucleotide (FAD)-binding motifs, nicotinamide adenine dinucleotide (NAD)-binding motifs, and 6-10 transmembrane domains, with isoelectric points between 6.73 and 9.46, and molecular weights between 67.2 and $79.9 \mathrm{kD}$. CjFRO1 and $C j F R O 2$ were predominantly found in the aboveground parts of $C$. junos, with $C j F R O 1$ highly expressed in leaves, and $C j F R O 2$ largely expressed in stems and leaves. $\mathrm{CjFRO3}$ was less expressed in roots, stems, and leaves. $\mathrm{CjFRO4}$ and $\mathrm{CjFRO5}$ were predominately found in roots. Under iron-deficient conditions, $C j F R O 4$ was significantly and specifically increased in the roots of $C$. junos, whereas CjFRO1 was upregulated in the roots and leaves.
\end{abstract}

Iron is an essential element for plant growth, which is involved in numerous vital biological processes, including chlorophyll biosynthesis, photosynthetic electron transfer, DNA synthesis, and nitrogen reduction. Although abundant in soil, iron is one of the most common nutrients to limit plant growth and development because of its particularly low solubility in aerobic soils at neutral and alkaline $\mathrm{pH}$ (Mimmo et al., 2014). Based on their mechanism for Fe acquisition, plants can be categorized into strategy I or strategy II plants (Eide et al., 1996; Robinson et al., 1999; Romheld, 1987; Romheld and Marschner, 1986). Strategy II plants include grasses with roots that secrete compounds known as phytosiderophores (PS), which chelate $\mathrm{Fe}^{3+}$ from the rhizosphere. Subsequently, the yellow stripe 1 carrier protein in the plasmalemma introduced the $\mathrm{Fe}^{3+}$-PS complex into the root cell. By contrast, strategy I plants are all dicots and nongraminaceous monocots, and the acquisition of $\mathrm{Fe}^{3+}$ occurs in reducing reaction processes: 1) proton excretion via a selfphosphalated type of adenosine triphosphatease, thus acidifying the surrounding soil to increase iron solubility; 2) reduction of $\mathrm{Fe}^{3+}$ to $\mathrm{Fe}^{2+}$ via $\mathrm{FRO}$; and 3) transport of $\mathrm{Fe}^{2+}$ via ironregulated transporter through the plasmalemma membrane.

The FRO protein is a key enzyme for $\mathrm{Fe}^{3+}$ acquisition from soil, which has been confirmed through its functionality for chlorosis tolerance and RNA-interference in plants (Gama et al., 2017; Lee et al., 2016; Martínez-Cuenca et al., 2016).

Received for publication 1 June 2017. Accepted for publication 4 Aug. 2017. This study was supported by the National Modern Agriculture (Citrus) Industry System of Special Funds (CARS-27) and the Chongqing Social Undertakings and livelihood Protection Science and Technology Innovation project (cstc206shms-ztzx 8000).

${ }^{1}$ Corresponding author. E-mail: chenshanchun@cric.cn.
The first FRO gene was identified in the model plant Arabidopsis thaliana (Robinson et al., 1999). Further FRO genes have been reported in a variety of plants such as Arachis hypogaea (Ding et al., 2009), Cucumis sativus (Waters et al., 2007), Lotus japonicus (Klein et al., 2012), Malus xiaojinensis (Wu et al., 2012), Medicago truncatula (Andaluz et al., 2009; Orozco-Mosqueda et al., 2012), Oryza sativa (Gross et al., 2003), Pisum sativum (Waters et al., 2002), and Solanum lycopersicum (Kong et al., 2013; Li et al., 2004). Eight FROs have been described in the genome of $A$. thaliana and shown to exhibit tissue-specific expression (Mukherjee et al., 2006; Wu et al., 2005). FRO family members regulate metal ion homeostasis in different locations in the plant. AtFRO2 is mainly expressed in epidermal cells of roots where it participates in the reduction of $\mathrm{Fe}^{3+}$ to $\mathrm{Fe}^{2+}$ in the rhizosphere. AtFRO3 is predominantly expressed in the vascular cylinder of roots where it might be involved in the transport of Fe. AtFRO7 is located in the chloroplast membrane, retaining iron homeostasis in the chloroplast (Connolly et al., 2003; Jeong et al., 2008; Jeong and Connolly, 2009; Mukherjee et al., 2006). Furthermore, FROs have been reported to have different regulatory mechanisms, although their overexpression in plants can rescue lime-induced chlorosis (Connolly et al., 2003; Li et al., 2011). AtFRO2 has been reported to be positively regulated by transcriptional factor FIT (FER-like iron-deficiency-induced transcription factor), and POPEYE (bHLH047) has been shown to upregulate the expression of AtFRO3 (Hindt and Guerinot, 2012; Long et al., 2010).

The local Citrus junos cultivar Ziyang Xiangcheng was reported to be tolerant to lime-induced iron chlorosis in both field and laboratory experiments, and it could efficiently uptake iron from calcareous soils. Until now, only CjFROI (GenBank 
no. DQ985810) had been cloned from C. junos. To explore whether additional FRO genes were present and functional in the $C$. junos genome, an in silico search was conducted for genes that encode FRO functions. With the exception of CjFRO1, the results showed that at least four additional novel genes were present, which indicate features of characterized FRO proteins. These features include the existence of highly conserved FAD-binding motif, NAD-binding motif, transmembrane regions, and oxidoreductase signature motifs (Andaluz et al., 2009; Mukherjee et al., 2006). In addition, $C j F R O$ gene expression was confirmed via real-time PCR in different tissues and in conditions of Fe sufficiency and deficiency. The identification of the $C j F R O$ gene family enables an improved understanding of Fe uptake, translocation, distribution, and usage mechanisms in citrus plants.

\section{Materials and Methods}

IN SILICO SEARCH AND IDENTIFICATION OF FRO GENE COPIES. To identify unknown FRO members, a homology Basic Local Alignment Search Tool (BLAST) search was performed, employing the previously identified CjFRO1 gene (GenBank no. DQ985810). The BLASTs were conducted in the database of the Citrus sinensis Genome Sequencing Resources (Xu et al., 2012). Based on the predicted cDNA sequences of FROs in the database, sense and antisense primers for each $C j F R O$ were designed, and the coding sequences were amplified from the total RNA of 1-year-old 'Ziyang Xiangcheng' seedlings via RT-PCR or RACE (634858; Clontech, Beijing, China). They were purified with the Biospin Gel Extraction Kit (BSC02M1; Bioer Technology Co., Hangzhou, China), subsequently cloned into a pMD19-T vector (6013; TaKaRa, Beijing, China), and sequenced. The sequences were predicted with the software packages Protparam (Gasteiger et al., 2005) and Pfam (Finn et al., 2016). Transmembrane domains of putative CjFRO proteins were predicted with the TMHMM program (Technical University of Denmark, 2017). Multiple alignments of sequences were performed using the ClustalX2.1 software package (Thompson et al., 2002), and the phylogenic tree was obtained with the software MEGA 5.0 (Tamura et al., 2011).

Plant materials AND growth Conditions. 'Ziyang Xiangcheng' seeds were germinated on wet filter paper in the dark for 1 week and were planted in jars with $1 / 2$ Hoagland's solution. The nutrient solution was replaced every 2 weeks. After 2 months, the seedlings were transferred into either iron-sufficient (50 $\mu \mathrm{M}$ FeEDTA) or iron-deficient ( $0 \mu \mathrm{M}$ FeEDTA) Hoagland's solutions for $2 \mathrm{~d}$ because of the FRO enzyme activity and gene expression could be induced after $2 \mathrm{~d}$ iron starvation in other plants (Mukherjee et al., 2006; Wu et al., 2012). Then, roots and leaves were harvested for iron starvation analysis.

One-year-old 'Ziyang Xiangcheng' seedlings were grown in the germplasm resources garden of the Citrus Research Institute (Chongqing, China). For analyzing the CjFROs expression in different tissues, the root tips $(1.5 \mathrm{~cm})$, stems (between fifth and sixth leaves), and the first fully developed leaves were collected from five plants.

RNA EXTRACTION. Total RNA was extracted with the RNA Extraction Kit (RN0902; Aidlab Biotechnologies Co., Beijing, China) and the RNA was treated with RNase-free DNase I to remove residual genomic DNA. RNA samples were run in a $1.2 \%$ agarose gel and stained with ethidium bromide to verify RNA quality. Each RNA sample was quantified with a spectrophotometer (NanoDrop 2000c; Thermo Fisher Scientific, Shanghai, China) at $260 \mathrm{~nm}$.

Full-Sequence Cloning of CJFROs. Full sequences were obtained via PCR using primer pairs listed in Supplemental Table 1. The PCR conditions were $95{ }^{\circ} \mathrm{C}$ for $1 \mathrm{~min} ; 94^{\circ} \mathrm{C}$ for $30 \mathrm{~s}, 58^{\circ} \mathrm{C}$ for $30 \mathrm{~s}, 72^{\circ} \mathrm{C}$ for $2 \mathrm{~min} 30 \mathrm{~s}$ for 40 cycles; followed by $72{ }^{\circ} \mathrm{C} 10 \mathrm{~min}$. The PCR products were cloned into the pMD19-T vector and transformed into competent Escherichia coli $\mathrm{DH} 5 \alpha$ cells. PCR confirmed positive clones were sequenced in the Beijing Genomics Institute (Shenzhen, China).

REAL-TIME PCR. cDNA was prepared from $1 \mu \mathrm{g}$ total RNA with the iScript ${ }^{\mathrm{TM}}$ cDNA Synthesis Kit following the manufacturer's instructions (170-8891; Bio-Rad Laboratories, Hercules, CA). Amplification with the primers in Supplemental
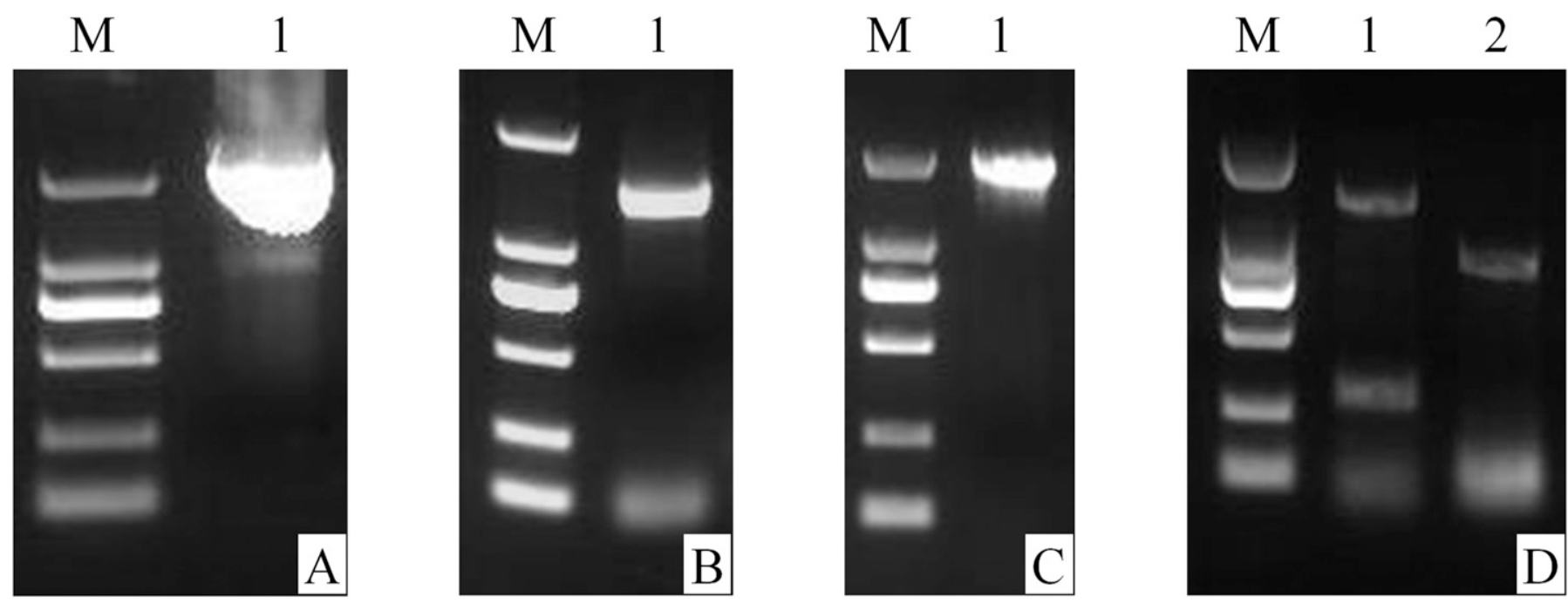

Fig. 1. Polymerase chain reaction (PCR) results of ferric chelate reductase genes of Citrus junos (CjFROs). CjFRO2 (A), 5' terminal PCR products of CjFRO3 (B), and CjFRO4 (C), were cloned with primers FROafS/FROafR, FROcfS2/FROcfR2, FROdfS/FROdfR, respectively. CjFRO5 (D) were cloned with two primer pairs FROedS/FROeqR3 (lane 1) and FROeqS3/FROefR (lane 2). The sequences of primer pairs could be found in Supplemental Table 1. Lane M was DNA Marker DL $2000(2000,1000,750,500,250$, and $100 \mathrm{bp})$. 
Table 1. Summarized features of the ferric chelate reductase genes of Citrus junos (CjFROs) from 'Ziyang Xiangcheng' according to their nucleic acid sequences and amino acid sequences.

\begin{tabular}{|c|c|c|c|c|c|c|c|}
\hline Name & Nucleic acids (no.) & Amino acids (no.) & MW $(\mathrm{kD})^{\mathrm{z}}$ & $\mathrm{pI}^{\mathrm{y}}$ & Transmembrane regions (no.) & Introns (no.) & Exons (no.) \\
\hline CjFROI & 2103 & 700 & 79.7 & 9.23 & 10 & 13 & 14 \\
\hline CjFRO3 & 2112 & 703 & 79.9 & 9.46 & 10 & 7 & 8 \\
\hline CjFRO5 & 2112 & 703 & 79.2 & 9.06 & 9 & 7 & 8 \\
\hline
\end{tabular}

${ }_{\mathrm{z}}^{\mathrm{z}}$ Molecular weight.

${ }^{\mathrm{y}}$ Isoelectric point.

Table 2 was performed using the SYBR Green PCR Master Mix (1725271; Bio-Rad Laboratories) and the iCycler iQ Multicolor Real-Time PCR Detection System (Bio-Rad Laboratories). The following protocol was used for amplification: $95^{\circ} \mathrm{C}$ for $1 \mathrm{~min}$; $94{ }^{\circ} \mathrm{C}$ for $10 \mathrm{~s}, 60^{\circ} \mathrm{C}$ for $15 \mathrm{~s}$, and $72^{\circ} \mathrm{C}$ for $15 \mathrm{~s}$, for 40 cycles. After completion of the amplification reaction, melt curves were compared to verify the amplification of a single peak. Each experiment was performed in at least three technical replications and three biological replicates were run per sample (using three independent sets of RNA). The data analysis used the $2^{-\Delta \mathrm{Ct}}$ method, and actin was used as a reference gene.

\section{Results}

IDENTIFICATION AND BIOINFORMATIC ANALYSIS OF CJFROs. The genome sequence of citrus was searched through BLASTx against the amino acid sequence of $C j F R O 1$. Five predicted sequences of C. sinensis (Cs3g01120, Cs9g19350, Cs5g06040, orange1.1t00399, and Cs9g19360) were found. Cs9g19350 corresponds to CjFRO1, and the other genes of 'Ziyang Xiangcheng' were designated as CjFRO2 (Cs3g01120), CjFRO3 (Cs5g06040), CjFRO4 (orange1.1t00399), and CjFRO5 (Cs9g19360). These revealed high-sequence identity among each other at the amino acid level (Supplemental Table 3).

Based on the predicted open reading frame sequences of the citrus genome database, sense and antisense primers were designed for all four novel CjFRO genes, and the cDNAs of these genes were amplified with the primers listed in the Supplemental Table 1, using total RNA that was isolated from 1-year-old 'Ziyang Xiangcheng' seedlings. Via RT-PCR, CjFRO2 was obtained with the primer pairs FROafS/FROasR of leaves, and $C j F R O 4$ was obtained with FROdfS/FROdfR of roots. For the remaining two FRO genes, it was difficult to obtain the whole open-reading-frame sequence. The upstream nucleic acid sequence of $C j F R O 5$ was obtained via PCR with the primer pair FROefS/FROeqR3, and the downstream sequence was obtained with the primer pair FROeqS3/FROefR. For $C j F R O 3$, the upstream nucleic acid sequence was obtained via PCR, using the primer pair FROcfS2/FROcfR2, and the downstream sequence was obtained via $3^{\prime}$ RACE with the primer pairs FROcfS3/3' RACE outer primer and the FROcfS4/3' RACE inner primer, which were subsequently merged (Fig. 1). These all contained the conserved features of $\mathrm{Fe}(\mathrm{III})$ chelate reductases described by Waters et al. (2002) and were classified into the FRO gene family of citrus. Analysis with the TMHMM program indicated that the deduced amino acid sequences of $C j F R O$ s contained six $(C j F R O 2$ and $C j F R O 4)$, nine (CjFRO5), or ten (CjFRO1 and CjFRO3) integral transmembrane domains. Most $C j F R O$ s belong to the basic protein excluding $C j F R O 2$, which is almost neutral with an isoelectric point of 6.73 (Table 1).

A neighbor-joining tree was constructed based on the deduced amino acid sequences of five $C j F R O$ s from citrus, eight AtFROs from A. thaliana (Wu et al., 2005), two MtFROs from M. truncatula (Andaluz et al., 2009), LjFRO1 from $L$. japonicus (Klein et al., 2012), LeFRO1 from S. lycopersicum (Kong et al., 2013), MxFRO from M. xiaojinensis (Wu et al., 2012), and PSFROI from P. sativum (Waters et al., 2002). The phylogenetic relationship of these five $C j F R O$ s could be clustered into four groups (Fig. 2). CjFRO4 and CjFRO5 were closer related to the well-characterized Fe(III) chelate rductase genes LeFRO1 from a herbaceous plant and MxFRO from a woody plant, respectively, all of which could be grouped with AtFRO2 and AtFRO3 of A. thaliana. CjFRO1 clustered with

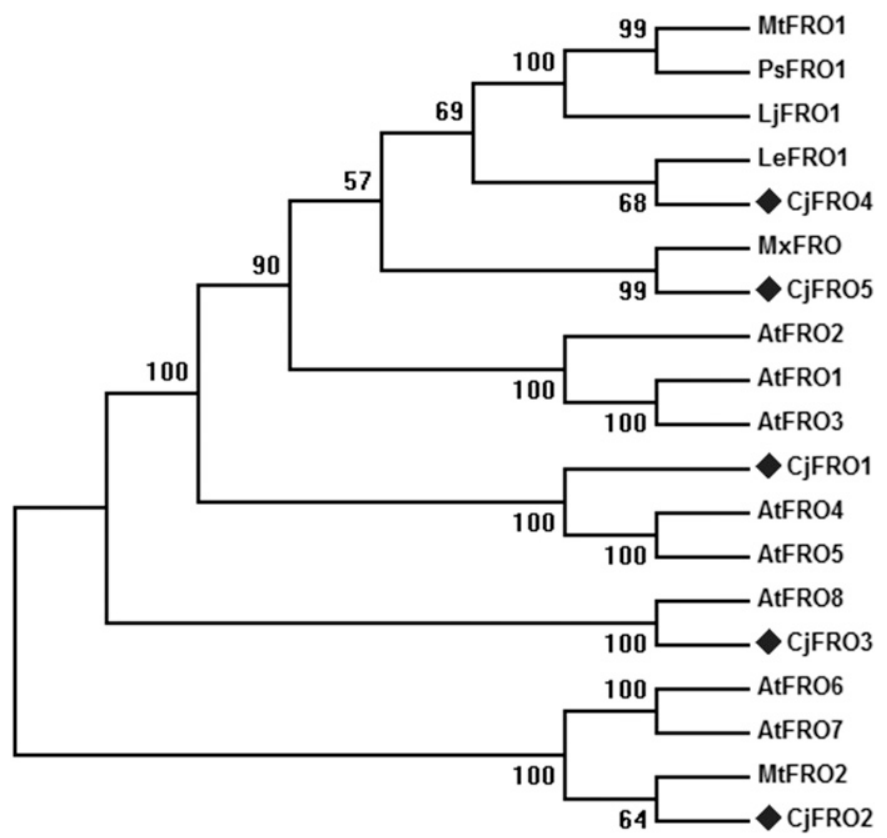

Fig. 2. Phylogenetic relationship of plant ferric chelate reductases (FRO). The complete predicted protein sequences of the five CjFROs in Citrus junos 'Ziyang Xiangcheng' were aligned with the other plant FROs through MEGA 5.0 (Tamura et al., 2011). AtFROs from Arabidopsis thaliana (AtFRO1, NM_100041; AtFRO2, NM_100040; AtFRO3, NM_102150; AtFRO4, NM_122303; AtFRO5, NM_122304; AtFRO6, NM_124351; AtFRO7, NM_124352; AtFRO8, NM_124395), MtFROs from Medicago truncatula (MtFRO1, AY439088; MtFRO2, XM_003594382), LjFRO1 from Lotus japonicus (JF290367), LeFRO1 from Solanum lycopersicum (AY224079), MxFRO from Malus xiaojinensis (EF577061), and PsFRO1 from Pisum sativum (AF405422). The bootstra $P$ values were obtained by the neighborjoining method and indicate the divergence of each branch. FROs from $C$. junos were highlighted with diamonds on the tree. 


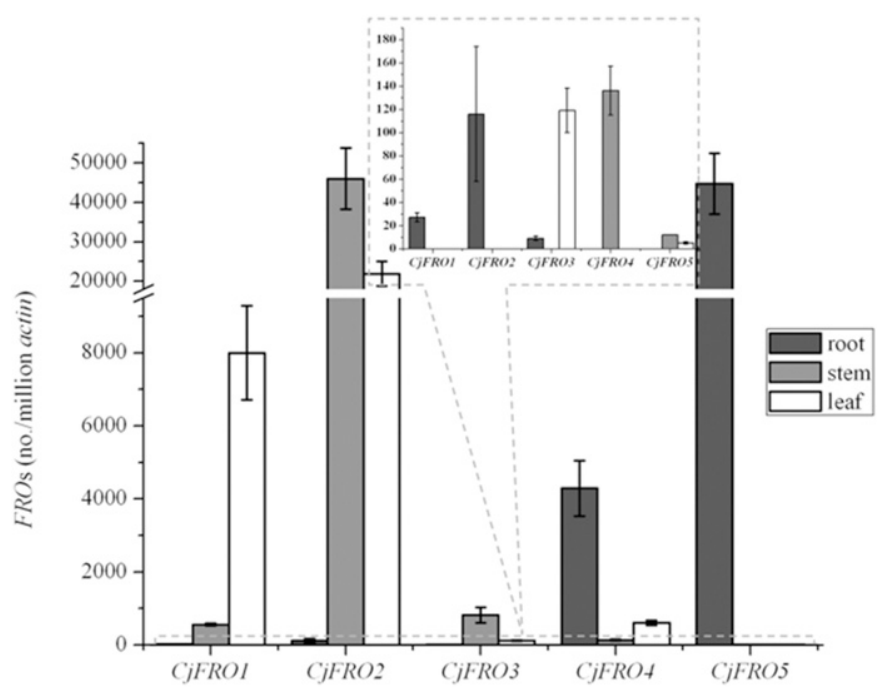

Fig. 3. The expression of ferric chelate reductase genes of Citrus junos (CjFROs) in different tissues of 1-year-old 'Ziyang Xiangcheng' seedlings. The transcript levels of $C j F R O \mathrm{~s}$ in citrus seedlings were detected using the real-time polymerase chain reaction. The relative expression level value was normalized to an internal standard actin. Each histogram represents the mean value of three independent experiments, and the vertical bars indicate SE. Dashed frame enlarges the results of the lower-expressed CjFROs in the dotted rectangle.

AtFRO4 and AtFRO5; CjFRO3 grouped with AtFRO8; CjFRO2 shared a group with AtFRO6 and AtFRO7.

EXPRESSION PROFILES OF CJFROS IN CITRUS TISSUES. Realtime PCR was performed to evaluate the expression of all five CjFROs in roots, stems, and leaves of 1-year-old 'Ziyang Xiangcheng' seedlings. The sense terminal primers and antisense terminal primers for real-time PCR (Supplemental Table 2) were designed in different exons, to avoid genomic DNA contamination. According to tissue expression (Fig. 3), CjFRO2 and CjFRO1 were expressed at higher levels in stems and leaves. Compared with $\mathrm{CjFRO2}$ expression in roots, its accumulation increased 396-fold in stems and 189-fold in leaves. CjFRO1 expression in stems and leaves was 21 -fold and 296-fold higher than in roots. However, CjFRO4 expression in roots was 31-fold higher than in stems and 7-fold higher than in leaves. CjFRO5 was a gene with a root-specific expression and was barely detectable by real-time PCR in stems and leaves. CjFRO3 showed lower expression in roots, stems, and leaves.

CJFROS Response to the Fe deficient condition. To analyze CjFROs expression in both Fe sufficiency and Fe deficiency conditions, citrus roots and leaves of 2-month-old seedlings, cultured in Hoagland's nutrient solution were tested via real-time PCR (Fig. 4). After $2 \mathrm{~d}$ of iron deficiency stress, CjFROI was upregulated 3.0-fold in roots and 1.9-fold in leaves. The expression $\mathrm{CjFRO2}$ and $\mathrm{CjFRO3}$ remained unchanged in roots and leaves (change fold $\leq 1.5$ ). CjFRO4 showed a specifically and significantly higher accumulation in roots (4.5-fold) under the Fe starvation and not changed in the leaves. CjFRO5 were not detectable in leaves and roots of 2-month-old seedlings under Fe-sufficient and Fe-starvation conditions.

\section{Discussion}

Online plant genomics offer greatly valuable databases for the identification and further characterization of genes that are involved in diverse cellular processes, such as Fe-starvation responses. Eight $F R O$ s had been found in the A. thialiana genome and six FROs in the M. truncatula according their genomic databases (Orozco-Mosqueda et al., 2012; Wu et al., 2005). Just like $A$. thialiana and $M$. truncatula, $C$. junos belongs to the plants using strategy I for $\mathrm{Fe}$ acquisition, which can reduce rhizospheric iron, thus increasing the bioavailability of iron. In the present in silico study, four putative sequences with high identity to $F R O$ s of other plants were identified and cloned (apart from CjFRO1, which was previously described.). All sequences described herein showed highly conserved characteristics of FRO proteins, such as FAD and NAD binding domains, transmembrane regions, and oxidoreductase signature motifs. FROs are present in various plant species and are
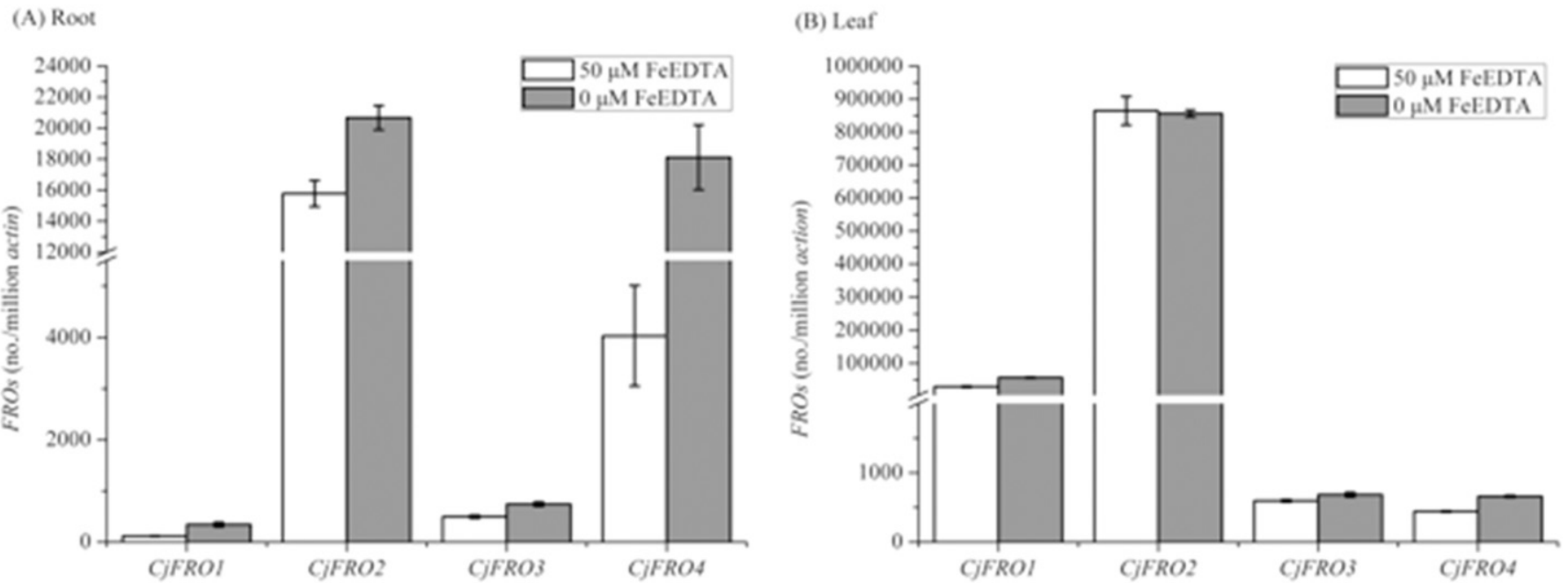

Fig. 4. Real-time polymerase chain reaction (PCR) analysis of Citrus junos ferric chelate reductase (FRO) gene-expression in root (left) and leaf (right) of ironsufficient and iron-deficient plants. The seedlings were initially cultured for 2 months in Hoagland's solution and then transferred to either iron-sufficient $(50 \mu \mathrm{M}$ FeEDTA) or iron-deficient ( $0 \mu \mathrm{M}$ FeEDTA) Hoagland's solution for $2 \mathrm{~d}$ of treatment. The $F R O$ relative expression level value was normalized to an internal standard actin. Each histogram represents the mean value of three independent experiments, and the vertical bars indicate SE. 
responsible for the reduction of $\mathrm{Fe}^{3+}$ to $\mathrm{Fe}^{2+}$, a more soluble form that can be absorbed by the roots. Gene expression and posttranslation of $\mathrm{FRO2}$ is positively regulated by carbon monoxide, nitric oxide, ethylene, and bHLH transcription factors on Fe-deficient conditions (Du et al., 2015; Hindt and Guerinot, 2012; Kabir et al., 2012; Yang et al., 2016; Yuan et al., 2008; Zhai et al., 2016).

In plants, AtFRO2 was the first FRO gene that was characterized (Robinson et al., 1999). Subsequently, seven other functional members of the same family have been identified in the model plant arabidopsis (Mukherjee et al., 2006; Wu et al., 2005). Similarly, six MtFROs were reported for M. truncatula (Orozco-Mosqueda et al., 2012) and five $C j F R O$ s for $C$. junos in our experiment. The reason why plants contain multiple $F R O$ genes in their genomes has already been summarized in excellent reviews: FROs are well known for their role in iron uptake by roots and furthermore, may play critical roles in the iron uptake by leaves as well as for iron homeostasis in chloroplasts and mitochondria (Jain et al., 2014; Jeong and Connolly, 2009). Moreover, FROs are not only found in strategy I plants, but also in strategy II plants, such as $O$. sativa that contains two $F R O$ genes (Ishimaru et al., 2007). The O. sativa FROI mutant revealed that $F R O$ might have a new function and might be involved in iron trafficking under Fe-toxic conditions (Siriphat et al., 2015).

In this study, multiple members of $F R O$ s in $C$. junos were found to be present in roots and shoots and are induced by $\mathrm{Fe}$ deficiency. These results agree with $F R O$ studies in A. thaliana, which demonstrated that genes such as $A t F R O 2$ and $A t F R O 3$ are mainly expressed in roots under low-Fe conditions, whereas AtFRO5, AtFRO6, AtFRO7, and AtFRO8 are exclusively found in shoots and not regulated by Fe supply (Wu et al., 2005). Furthermore, the expression of each gene is tissue specific; for example, AtFRO2 and AtFRO3 are mainly expressed in roots, whereas AtFRO5 and AtFRO6 were detected in shoots and flowers, and AtFRO7 in cotyledons and trichomes. AtFRO6 and AtFRO7 were located on plasma membranes and chloroplast membranes, respectively, whereas AtFRO8 was specifically expressed in the veins of leaves (Jeong et al., 2008; Wu et al., 2005). In C. junos, CjFRO4 and CjFRO5 were clustered with the well-characterized plant $F R O s$, which were highly or specificly expressed in the roots. CjFRO1-3 was clustered with the shoot-specific-expressed AtFROs (Fig. 2). The real-time PCR analysis for the five $C j F R O$ s confirmed the result of cluster grouping (Fig. 3). CjFRO4 accumulated significantly higher levels in roots under iron starvation (Fig. 4). The lack of CjFRO5 observation in leaves and roots was unexpected, although it was highly expressed in the roots of 1-year-old 'Ziyang Xiangcheng' seedlings. This phenomenon has been described in arabidopsis for AtFRO3, which was expressed at high levels in seedlings, but at lower levels in mature, soilgrown plants (Mukherjee et al., 2006).

In conclusion, at least five $F R O$ genes have been found within the citrus genome. Here, we detected the four novel genes CjFRO2, CjFRO3, CjFRO4, and CjFRO5, which had the transmembrane domain, FAD-binding domain, and NAD binding domain of ferric reductase. The genes CjFRO5 and $C j F R O 4$ were highly expressed in roots, whereas $C j F R O 2$ and $C j F R O 1$ were highly expressed in leaves. CjFRO3 had lower expression in roots, stems, and leaves. Fe stress significantly increased $\mathrm{CjFRO}_{4}$ expression in roots.

\section{Literature Cited}

Andaluz, S., J. Rodriguez-Celma, A. Abadia, J. Abadia, and A.F. Lopez-Millan. 2009. Time course induction of several key enzymes in Medicago truncatula roots in response to Fe deficiency. Plant Physiol. Biochem. 47:1082-1088.

Connolly, E.L., N.H. Campbell, N. Grotz, C.L. Prichard, and M.L. Guerinot. 2003. Overexpression of the FRO2 ferric chelate reductase confers tolerance to growth on low iron and uncovers posttranscriptional control. Plant Physiol. 133:1102-1110.

Ding, H., L. Duan, H. Wu, R. Yang, H. Ling, W.X. Li, and F. Zhang. 2009. Regulation of AhFRO1, an Fe(III)-chelate reductase of peanut, during iron deficiency stress and intercropping with maize. Physiol. Plant. 136:274-283.

Du, J., Z. Huang, B. Wang, H. Sun, C. Chen, H.Q. Ling, and H. Wu. 2015. SlbHLH068 interacts with FER to regulate the iron-deficiency response in tomato. Ann. Bot. 116:27-31.

Eide, D., M. Broderius, J. Fett, and M.L. Guerinot. 1996. A novel iron-regulated metal transporter from plants identified by functional expression in yeast. Proc. Natl. Acad. Sci. USA 93:56245628.

Finn, R.D., P. Coggill, R.Y. Eberhardt, S.R. Eddy, J. Mistry, A.L. Mitchell, S.C. Potter, M. Punta, M. Qureshi, A. Sangrador-Vegas, G.A. Salazar, J. Tate, and A. Bateman. 2016. The Pfam protein families database: Towards a more sustainable future. Nucleic Acids Res. 44: D279-D285.

Gama, F., T. Saavedra, S. Dandlen, A.d. Varennes, P.J. Correia, M. Pestana, and G. Nolasco. 2017. Silencing of the FRO1 gene and its effects on iron partition in Nicotiana benthamiana. Plant Physiol. Biochem. 114:111-118.

Gasteiger, E., C. Hooglant, A. Gattiker, S. Duvaud, M.R. Wilkins, R.D. Appel, and A. Bairoch. 2005. Protein identification and analysis tools in the ExPASy server, p. 571-607. In: J.M. Walker (ed.). The proteomics protocols handbook. Humana Press, Totowa, NJ.

Gross, J., R.J. Stein, A.G. Fett-Neto, and J.P. Fett. 2003. Iron homeostasis related genes in rice. Genet. Mol. Biol. 26:477-497.

Hindt, M.N. and M.L. Guerinot. 2012. Getting a sense for signals: Regulation of the plant iron deficiency response. Biochim. Biophys. Acta 1823:1521-1530.

Ishimaru, Y., S. Kim, T. Tsukamoto, H. Oki, T. Kobayashi, S. Watanabe, S. Matsuhashi, M. Takahashi, H. Nakanishi, S. Mori, and N.K. Nishizawa. 2007. Mutational reconstructed ferric chelate reductase confers enhanced tolerance in rice to iron deficiency in calcareous soil. Proc. Natl. Acad. Sci. USA 104:7373-7378.

Jain, A., G.T. Wilson, and E.L. Connolly. 2014. The diverse roles of FRO family metalloreductases in iron and copper homeostasis. Front. Plant Sci. 5:100.

Jeong, J., C. Cohu, L. Kerkeb, M. Pilon, E.L. Connolly, and M.L. Guerinot. 2008. Chloroplast Fe(III) chelate reductase activity is essential for seedling viability under iron limiting conditions. Proc. Natl. Acad. Sci. USA 105:10619-10624.

Jeong, J. and E.L. Connolly. 2009. Iron uptake mechanisms in plants: Functions of the FRO family of ferric reductases. Plant Sci. 176:709714.

Kabir, A.H., N.G. Paltridge, A.J. Able, J.G. Paull, and J.C.R. Stangoulis. 2012. Natural variation for Fe-efficiency is associated with upregulation of Strategy I mechanisms and enhanced citrate and ethylene synthesis in Pisum sativum L. Planta 235:14091419.

Klein, M.A., A.F. Lopez-Millan, and M.A. Grusak. 2012. Quantitative trait locus analysis of root ferric reductase activity and leaf chlorosis in the model legume, Lotus japonicus. Plant Soil 351:363-376.

Kong, D.Y., C.L. Chen, H.L. Wu, L. Ye, J.M. Li, and H.Q. Ling. 2013. Sequence diversity and enzyme activity of ferric-chelate reductase LeFRO1 in tomato. J. Genet. Genomics 40:565-573.

Lee, S.R., M.M. Oh, and S.A. Park. 2016. Ferric-chelate reductase activity is a limiting factor in iron uptake in spinach and kale roots. Hort. Environ. Biotechnol. 57:462-469. 
Li, L., X. Cheng, and H. Ling. 2004. Isolation and characterization of $\mathrm{Fe}(\mathrm{III})$-chelate reductase gene LeFROI in tomato. Plant Mol. Biol. 54:125-136.

Li, L.Y., Q.Y. Cai, D.S. Yu, and C.H. Guo. 2011. Overexpression of AtFRO6 in transgenic tobacco enhances ferric chelate reductase activity in leaves and increases tolerance to iron-deficiency chlorosis. Mol. Biol. Rpt. 38:3605-3613.

Long, T.A., H. Tsukagoshi, W. Busch, B. Lahner, D.E. Salt, and P.N. Benfey. 2010. The bHLH transcription factor POPEYE regulates response to iron deficiency in Arabidopsis roots. Plant Cell 22:2219-2236.

Martínez-Cuenca, M.R., A. Quiñones, and M.Á. Forner-Giner. 2016. Screening of 'King' mandarin (Citrus nobilis Lour. $\times$ Poncirus trifoliata (L.) Raf.) hybrids as citrus rootstocks tolerants to iron chlorosis. Sci. Hort. 198:61-69.

Mimmo, T., D. Del Buono, R. Terzano, N. Tomasi, G. Vigani, C. Crecchio, R. Pinton, G. Zocchi, and S. Cesco. 2014. Rhizospheric organic compounds in the soil-microorganism-plant system: Their role in iron availability. European J. Soil Sci. 65:629-642.

Mukherjee, I., N.H. Campbell, J.S. Ash, and E.L. Connolly. 2006. Expression profiling of the Arabidopsis ferric chelate reductase $(F R O)$ gene family reveals differential regulation by iron and copper. Planta 223:1178-1190.

Orozco-Mosqueda, M.C., G. Santoyo, R. Farias-Rodriguez, L. MacasRodriguez, and E. Valencia-Cantero. 2012. Identification and expression analysis of multiple FRO gene copies in Medicago truncatula. Genet. Mol. Res. 11:4402-4410.

Robinson, N.J., C.M. Procter, E.L. Connolly, and M.L. Guerinot. 1999. A ferric-chelate reductase for iron uptake from soils. Nature 397:694-697.

Romheld, V. 1987. Different strategies for iron acquisition in higher plants. Physiol. Plant. 70:231-234.

Romheld, V. and H. Marschner. 1986. Evidence for a specific uptake system for iron phytosiderophores in roots of grasses. Plant Physiol. 80:175-180.

Siriphat, R., R. Vinitchan, S. Chatree, P. Supaporn, T. Somvong, K. Ratchanee, and V. Apichart. 2015. Forward screening for seedling tolerance to $\mathrm{Fe}$ toxicity reveals a polymorphic mutation in ferric chelate reductase in rice. Rice 8:3.

Tamura, K., D. Peterson, N. Peterson, G. Stecher, M. Nei, and S. Kumar. 2011. MEGA5: Molecular evolutionary genetics analysis using maximum likelihood, evolutionary distance, and maximum parsimony methods. Mol. Biol. Evol. 28:2731-2739.
Technical University of Denmark. 2017. TMHMM Server v. 2.0. 4 July 2017. <http://www.cbs.dtu.dk/services/TMHMM-2.0/>.

Thompson, J.D., T.J. Gibson, and D.G. Higgins. 2002. Multiple sequence alignment using ClustalW and ClustalX. Curr. Protoc. Bioinformatics Chapter 2:Unit 2.3.

Waters, B.M., D.G. Blevins, and D.J. Eide. 2002. Characterization of FRO1, a pea ferric-chelate reductase involved in root iron acquisition. Plant Physiol. 129:85-94.

Waters, B.M., C. Lucena, F.J. Romera, G.G. Jester, A.N. Wynn, C.L. Rojas, E. Alcantara, and R. Perez-Vicente. 2007. Ethylene involvement in the regulation of the $\mathrm{H}^{+}$-ATPase CsHAl gene and of the new isolated ferric reductase $C s F R O 1$ and iron transporter CsIRT1 genes in cucumber plants. Plant Physiol. Biochem. 45:293301.

Wu, H., L. Li, J. Du, Y. Yuan, X. Cheng, and H.Q. Ling. 2005. Molecular and biochemical characterization of the Fe(III) chelate reductase gene family in Arabidopsis thaliana. Plant Cell Physiol. 46:1505-1514.

Wu, T., H.T. Zhang, Y. Wang, W.S. Jia, X.F. Xu, X.Z. Zhang, and Z.H. Han. 2012. Induction of root $\mathrm{Fe}(111)$ reductase activity and proton extrusion by iron deficiency is mediated by auxin-based systemic signalling in Malus xiaojinensis. J. Expt. Bot. 63:859-870.

Xu, Q., L.L. Chen, X.A. Ruan, D.J. Chen, A.D. Zhu, C.L. Chen, D. Bertrand, W.B. Jiao, B.H. Hao, M.P. Lyon, J.J. Chen, S. Gao, F. Xing, H. Lan, J.W. Chang, X.H. Ge, Y. Lei, Q. Hu, Y. Miao, L. Wang, S.X. Xiao, M.K. Biswas, W.F. Zeng, F. Guo, H.B. Cao, X.M. Yang, X.W. Xu, Y.J. Cheng, J. Xu, J.H. Liu, O.J. Luo, Z.H. Tang, W.W. Guo, H.H. Kuang, H.Y. Zhang, M.L. Roose, N. Nagarajan, X.X. Deng, and Y.J. Ruan. 2012. The draft genome of sweet orange (Citrus sinensis). Nat. Genet. 45:59-66.

Yang, L., J. Ji, H. Wang, K.R. Harrisshultz, E.F. Abd Allah, Y. Luo, Y. Guan, and X. Hu. 2016. Carbon monoxide interacts with auxin and nitric oxide to cope with iron deficiency in Arabidopsis. Front. Plant Sci. 7:112.

Yuan, Y., H. Wu, N. Wang, J. Li, W. Zhao, J. Du, D. Wang, and H.Q. Ling. 2008. FIT interacts with AtbHLH38 and AtbHLH39 in regulating iron uptake gene expression for iron homeostasis in Arabidopsis. Cell Res. 18:385-397.

Zhai, L., D. Xiao, C. Sun, T. Wu, Z. Han, X. Zhang, X. Xu, and Y. Wang. 2016. Nitric oxide signaling is involved in the response to iron deficiency in the woody plant Malus xiaojinensis. Plant Physiol. Biochem. 109:515-524. 
Supplemental Table 1. Primers used for full cDNA sequence cloning of ferric chelate reductase genes of Citrus junos (CjFROs). FROafS and FROafR were used for $C j F R O 2$ PCR. Primer pairs FROcfS2/FROcfR2 were used to obtain the upstream sequence of $C j F R O 3$, FROcfS3/3' RACE outer primer and FROcfS4/3' RACE inner primer for the downstream sequence of CjFRO3. FROdfS and FROdfR were used for CjFRO4 PCR. Primer pairs FROefS/FROeqR3 and FROeqS3/FROefR were used to obtain the upstream and downstream sequences of CjFRO5, respectively.

\begin{tabular}{|c|c|c|}
\hline Genes & Sense primers & Antisense primers \\
\hline$\overline{C j F R O 2}$ & FROafS: 5' -ATGTTGGAACTGACGGGACTT-3' & FROafR: 5'-CTACAGATCAAAACTATGGCTGTGG-3' \\
\hline \multirow{2}{*}{ CjFRO3 } & FROcfS3: 5' -AGCATGCAGGCTTAAAGTTCACT-3' & $\begin{array}{l}\text { 3' RACE outer primer: 5' -TACCGTCGTTCCACTAGTG } \\
\text { ATTT-3' }\end{array}$ \\
\hline & FROcfS4: 5' -CGAGTATTTCCAAATTTCAGTGGCA-3' & $\begin{array}{l}3^{\prime} \text { RACE inner primer: 5'-CGCGGATCCTCCACTAGTG } \\
\text { ATTTCACTATAGG-3' }\end{array}$ \\
\hline CjFRO4 & FROdfS: 5'-ATGCTGGTGAAAGGCCCACT-3' & FROdfR: 5' -TCACCAGCTAAAGCTGATAGATTC-3' \\
\hline CjFRO5 & FROeqS3: 5' -CCATTTTACAGGGCACGACAT-3' & FROefR: 5' -TCACCAGCTAAAGGCTATGGATT-3' \\
\hline
\end{tabular}

$\overline{\mathrm{PCR}}=$ polymerase chain reaction; $\mathrm{RACE}=$ rapid amplification of cDNA ends.

Supplemental Table 2. Primers used for analyzing the expression of Citrus junos ferric chelate reductase genes (CjFROs) in different tissues and responding to the iron starvation.

\begin{tabular}{|c|c|c|c|}
\hline Genes & Sense primers & Antisense primers & cDNA length $(\mathrm{bp})$ \\
\hline CjFRO2 & 5'-AGGCACCCTTTACTACCTCCTAC-3' & 5'-GACAAGACTTCCCTTCGTTGATA-3' & 181 \\
\hline CjFRO3 & 5'-CCAGCTTTGAGATTCTGTTGGT-3' & 5' - GGTACTTGAGTTGCCATGTGTT-3' & 135 \\
\hline CjFRO5 & 5'-CCATTTTACAGGGCACGACAT-3' & 5' - ATTATTGGCCAAAGAGAGCAGC-3' & 100 \\
\hline actin & 5'-CATCCCTCAGCACCTTCC-3' & 5' -CCAACCTTAGCACTTCTCC-3' & 198 \\
\hline
\end{tabular}

Supplemental Table 3. Distributions of percentage protein sequence identity for pairs of Citrus junos ferric chelate reductases (CjFROs).

\begin{tabular}{|c|c|c|c|c|c|}
\hline & CjFRO1 & $\mathrm{CjFRO2}$ & $\mathrm{CjFRO3}$ & CjFRO4 & $\mathrm{CjFRO5}$ \\
\hline$\overline{\mathrm{CjFRO}}$ & & 35 & 30 & 58 & 54 \\
\hline $\mathrm{CjFRO} 2$ & & & 32 & 35 & 33 \\
\hline $\mathrm{CjFRO} 3$ & & & & 32 & 31 \\
\hline CjFRO4 & & & & & 69 \\
\hline CjFRO5 & & & & & \\
\hline
\end{tabular}

